\title{
Proteomics profiling of human synovial fluid suggests global increased protein interplay in early- osteoarthritis (OA) and lost in late-stage OA
}

Neserin Ali $^{1 \neq}$, Aleksandra Turkiewicz ${ }^{1}$, Velocity Hughes ${ }^{1}$, Elin Folkesson ${ }^{1,3}$, Jon Tjörnstand ${ }^{2}$, Paul Neuman ${ }^{2}$, Patrik Önnerfjord ${ }^{3 *}$, Martin Englund ${ }^{1 *}$

* shared senior authors

${ }^{1}$ Clinical Epidemiology Unit, Orthopedics, Department of Clinical Sciences Lund, Lund University, Lund, Sweden

${ }^{2}$ Department of Orthopedics, Skåne University Hospital, Sweden

${ }^{3}$ Molecular Skeletal Biology, Section for Rheumatology, Department of Clinical Sciences Lund, Lund University, Lund, Sweden

\section{Corresponding author}

Neserin ali

Email: neserin.ali@med.lu.se

Phone: +46735304981

Running title: Proteomics profiling of human synovial fluid in knee Osteoarthritis

Abbreviations: Ethanol alcohol (EtOH), Sodium acetate (NaAc), Nano liquidchromatography (nLC), Automatic Gain Control (AGC) and Liver X Receptor-Retinoid X Receptor (LXR/RXR) 


\section{Abstract}

2 The underlying molecular mechanisms in osteoarthritis (OA) development are largely

3 unknown. This study explores the proteome and the pairwise interplay of proteins on a global

4 level in synovial fluid from patients with late-stage knee OA (arthroplasty), early knee OA

5 (arthroscopy due to degenerative meniscal tear) and from deceased controls without knee OA.

6 Synovial fluid samples were analyzed using state-of-the-art mass spectrometry with data-

7 independent acquisition. The differential expression of the proteins detected was clustered and

8 evaluated with data mining strategies and a multilevel model. Group-specific slopes of

9 associations were estimated between expressions of each pair of identified proteins to assess

10 the co-expression (i.e. interplay) between the proteins in each group.

11 More proteins were increased in early-OA vs controls than late-stage OA vs controls. For

12 most of these proteins, the fold changes between late-stage OA vs controls and early stage OA

13 vs controls were remarkably similar suggesting potential involvement in the OA process.

14 Further, for the first time this study illustrated distinct patterns in protein co-expression

15 suggesting that the global interplay between the protein machinery is increased in early-OA

16 and lost in late-stage OA. Further efforts should probably focus on earlier stages of the

17 disease than previously considered.

\section{Introduction}

20 Osteoarthritis $(\mathrm{OA})$, a degenerative joint disease, is one of the most common chronic health

21 conditions and a leading cause of pain and disability among adults ${ }^{1,2}$. Signs of OA are present

22 in 5 percent of the population between the age of 35-54 years, and the occurrence of the 
23 disease increases with age ${ }^{3,4}$. Despite the high burden of OA, its pathogenesis remains

24 unclear, and no true biological treatment exists on the global market ${ }^{2,5}$.

25 The classic diagnosis of OA relies on the presence of clinical symptoms in combination with 26 radiographic findings of joint degeneration, which are seen at a relatively late stage of the 27 disease ${ }^{6}$. Thus, the search for reliable biomarkers to diagnose OA and to assess its risk of 28 progression is at full speed ${ }^{7}$. Discovery of OA biomarkers would be useful in identifying 29 individuals with early disease, e.g. for recruitment to clinical trials, as well as for 30 supplementary monitoring of effects of therapies aimed at disease modification. Finding good 31 biomarkers representing the progression of OA goes hand in hand via exploring the 32 pathogenesis. Former studies to disentangle the molecular pathogenesis of OA or to identify 33 biomarkers have typically focused on specific molecules or signalling pathways ${ }^{7-9}$. However, 34 the "single biomarker strategy" has over and over again shown its lack of sensitivity and 35 specificity for progression of the disease ${ }^{9-11}$. In contrast, the global crosstalk between 36 molecules could be very informative in order to explore and gain deeper insights into the 37 molecular pathogenesis of $\mathrm{OA}^{12}$. While a number of different omics studies have been 38 carried out $^{13-19}$, they were limited by the technologies used or availability of biological 39 samples. Therefore, the aim of this study was to explore disease stages of OA using a global 40 discovery approach based on state-of-the-art mass spectrometry (MS) instrumentation and 41 methodology. The disease stages were represented by human synovial fluid from 1) patients 42 with late-stage knee OA, 2) patients with degenerative meniscus tears (indicative of early43 stage OA), and 3) controls without OA, to explore the differential expression and the pairwise 44 interplay of proteins measured. 


\section{Experimental procedures}

46

47

48

49

\section{Materials}

The micro bicinchoninic acid (BCA) protein assay kit and Nanosep® 30K Omega Centrifugal Devices were purchased from Pall Life Sciences (Ann Arbor MI, USA) and SOLA $\mu^{\mathrm{TM}}$ Solid Phase Extraction (SPE) HRP 2mg/1ml 96-well plates were purchased from Thermo Fisher Scientific (Rockford IL, USA). Trypsin (sequencing grade) was purchased from Promega Corporation (Madison, WI, USA). Calcium chloride, formic acid (FA), hydrochloric acid, and ammonium acetate were purchased from Merck (Darmstadt, Germany). Dithiothreitol (DTT) and iodoacetamide were purchased from Sigma-Aldrich (St. Louis, MO, USA). Acetonitrile (ACN) was purchased from Sigma-Aldrich (St. Louis MO, USA).

\section{Experimental design and statistical rationale}

Synovial fluid was sampled from 3 different types of subjects from the MENIX biobank at Skåne University Hospital, Lund: $i$ ) end-stage medial compartment knee OA patients undergoing total knee arthroplasty ( $\mathrm{n}=11$ [3 men and 8 women], age range 55-80 years), the arthroplasty synovial fluid selected for this study will hereafter be called late-stage OA synovial. ii) knee arthroscopy patients who had a typical degenerative meniscal tear ( $\mathrm{n}=7$ [3 men and 4 women], age range 50-64 years), the arthroscopy synovial fluid selected for this study will hereafter be called early-stage OA synovial. and iii) human deceased donors (controls), without evidence of tibiofemoral OA or known clinical knee OA ( $\mathrm{n}=13$ [5 men and 8 women] age range 19-79 years). All human deceased donor samples were obtained within $48 \mathrm{~h}$ post-mortem, and the specimens were frozen at $-80^{\circ} \mathrm{C}$ within $2 \mathrm{~h}$ of extraction. To be eligible as controls, the donor menisci were required to be macroscopically intact. Further, the femoral cartilage (the load bearing region) from the medial compartment of the same donors (also in the biobank) were inspected and required the cartilage to be macroscopically intact. The synovial fluid samples were obtained by transcutaneous aspiration immediately prior to 
70 the respective procedures. The donor synovial fluid selected for this study will hereafter be

71 called control synovial. All synovial fluids were centrifuged at $1800 \mathrm{rpm}$ for $10 \mathrm{~min}$ and

72 supernatants and pellets were separately frozen and stored at $-80^{\circ} \mathrm{C}$. The synovial fluid

73 samples' selection criteria from the biobank were also based on no visual signs of blood

74 contamination. Informed consent was taken before collecting the samples for bio-banking.

75 The sample collection and analysis has been proven by the ethical review committee of Lund

76 University (Durs: 2015/39;2016/865; 2019/3239) and carried out in accordance with relevant

77 guidelines and regulations.

78 Sample preparation

79 The total protein content in the synovial fluid sample was determined using a BCA protein

80 assay kit. From each sample $50 \mu \mathrm{L}$ synovial fluid were mixed with $10 \mu \mathrm{L}$ MS-safe proteinase

81 inhibitor cocktail and $10 \mu \mathrm{L}$ hyaluronidase $10 \mu \mathrm{L} / \mu \mathrm{g}$ protein and further incubated for $3 \mathrm{~h}$ at

$8237^{\circ} \mathrm{C}$. Four out of the 7 samples in the early OA samples were diluted with saline solution

83 during sampling of synovial fluid; these samples were adjusted to the mean total protein

84 content calculated from the remaining 3 samples in that group. All samples were depleted of

85 the 7 most abundant proteins with the multiple affinity removal system (MARS Hu7 spin

86 cartridge) according to the manufacturers protocol (Agilent Technologies). Samples were

87 further reduced using $4 \mathrm{mM}$ DTT, with shaking at $+56^{\circ} \mathrm{C}$ for $30 \mathrm{~min}$ and alkylated using 16

$88 \mathrm{mM}$ iodoacetamide for $1 \mathrm{~h}$ at room temperature in the dark. In order to remove residual salts,

89 the samples were precipitated with 1:9 volume $95 \% \mathrm{EtOH}$ (with $50 \mathrm{mM} \mathrm{NaAc}$ ) at $4{ }^{\circ} \mathrm{C}$

90 overnight. The precipitated samples were centrifuged and the supernatants were removed

91 from the pellets. The samples were dissolved in $0.1 \mathrm{M}$ ammonium bicarbonate and digested

92 with sequencing grade trypsin (Promega) at a protease/protein ratio of 1:50, overnight at

$9337^{\circ} \mathrm{C}$. Following digestion, samples were cleaned up with a 30kDa filter (Pall Life Sciences)

94 and the flow-through was desalted with $\mathrm{C} 1896$ well plates $\left(\mathrm{SOLA} \mu^{\mathrm{TM}}\right.$ Thermo Fisher 
95 Scientific). The samples were further spiked with iRT peptides before analyses with mass

96 spectrometry.

97 Instrumentation and data analysis

98 The samples were analyzed with an EASY-nLC 1000 (Thermo Scientific) coupled to a

99 Thermo Scientific Q-Exactive HFX ${ }^{\mathrm{TM}}$ mass spectrometer using data-independent acquisition

100 (DIA). For liquid chromatography, mobile phase A consisted of water containing $0.1 \%$ formic

101 acid (FA) and mobile phase B consisted of acetonitrile (ACN) containing $0.1 \%$ FA. Peptides

102 were loaded on an Acclaim PepMap® 100 nanoViper pre-column (Thermo Scientific, C18, 3

$103 \mu \mathrm{m}$ particles, $75 \mu \mathrm{m}$ i.d. $2 \mathrm{~cm}$ long) at $300 \mathrm{~nL} / \mathrm{min}$ for $125 \mathrm{~min}$ using $95 \%$ mobile phase A.

104 The peptides were separated on a PepMap® RSLC C18 analytical column (Thermo

105 Scientific, C18, $2 \mu \mathrm{m}$ particles, $75 \mu \mathrm{m}$ i.d. $25 \mathrm{~cm}$ long) at $300 \mathrm{~nL} / \mathrm{min}$ using an $\mathrm{ACN} /$ formic

106 acid gradient consisting of an initial step of 5-7\% B over 5 min followed by 7-20\% B over 85

$107 \mathrm{~min}, 20-30 \%$ B over $20 \mathrm{~min}, 30-90 \%$ B over $5 \mathrm{~min}$, held at $90 \%$ B for $5 \mathrm{~min}$ and then

108 equilibrated for $15 \mathrm{~min}$ at $3 \% \mathrm{~B}$, Separation was performed at $45^{\circ} \mathrm{C}$ and the total acquisition

109 time was 125 min. DIA settings: method duration $125 \mathrm{~min}$, full scan resolution 120 000, scan

110 range 350-1650 m/z. AGC target 3.0e6, maximum injection time $100 \mathrm{~ms}$, Orbitrap resolution

11145 000, AGC 3.0e5 with a variable isolation window 30/ 26/22/ 20/ 18/ 20/ 19/ 20/ 21/ 23/

$11224 / 26 / 31 / 32 / 37 / 40 / 53 / 66 / 99 / 574$ m/z, and normalized collision energy 27 eV. Data-

113 dependent acquisition (DDA) settings: method duration $125 \mathrm{~min}$, mass range 350-1650 m/z.,

114 full MS scan resolution 120 000, AGC 3e6, maximum injection time 20 ms, Orbitrap

115 resolution 15000 , AGC target $1.0 \mathrm{e} 5$, maximum injection $20 \mathrm{~ms}$, normalized collision energy

$11627 \mathrm{eV}$. The MS raw data were further analyzed with Spectronaut ${ }^{\mathrm{TM} P u l s a r}$ software (version

117 12.0.20491.15, Biognosys AG, Switzerland) for protein identification and quantitative data

118 extraction. In total 118 runs were used from both the DDA and DIA files were converted to

119 HTRMS format using HTRMS Converter (Biognosys AG, Switzerland) to generate the 
120 spectral library. The human protein fasta files were downloaded from the uniprot database

121 (20190416). Default settings were used with additional modifications: cysteine

122 carbamidomethylation was used as a fixed modification, and deamination, pyro-glutamic acid

123 (N-term Glu to pyroglutamic acid), methionine oxidation, hydroxyproline and acetylation

124 were used as variable modifications. Using Trypsin/P as the specific digestion type with

125 maximum of 2 miss cleavages. A subsequent protein search was conducted in Spectronaut ${ }^{\mathrm{TM}}$

126 Pulsar using the recently created spectral library and the same human database as background

127 proteome. Precursor quantitation was performed at MS2 level, and area under the curve was

128 used as quantitation type.

\section{Statistical analysis}

130 The quantitative data were extracted using Spectronaut ${ }^{\mathrm{TM} P u l s a r}$ software. The differential

131 expression of proteins between the different groups was analyzed using a linear mixed effects

132 model with the sample group, protein type and their interaction as independent variables and

133 subject as a random effect to account for clustering of proteins within an individual, and

134 transformed differential expression as the outcome ${ }^{20}$.The protein expression was transformed

135 using logarithm with base 2 before the analysis. Further, the model was adjusted for age, sex

136 and body mass index. Only proteins that had a maximum of one missing value in the early-

137 stage OA group, two missing values in the late-stage OA group and two missing values in the

138 control group were included, to enable enough samples for estimation. Residual diagnostics

139 confirmed adequate model fit. As the sample size of the early-stage OA group was lower than

140 the others due to sample availability, and also because this group had the highest amount of

141 missing data on protein differential expression, a sensitivity analysis where performed where

142 the early stage OA group was, allowing a larger set of proteins to be included in this analysis,

143474 proteins (compared to 406 proteins when including all three groups). 


\section{Data visualization and presentation}

\section{$145 \quad$ Clustering}

146 Principal component analysis (PCA) was used to cluster the samples based on their protein 147 expression data, after filtering out proteins based on missing values (max one missing value

148 for the early-stage OA group and two for the control and late-stage OA groups, as described

149 above) 406 proteins were included in the analysis. PCA pre-processing included a Pareto

150 scaling using "RFmarkerDetector::paretoscale" functions in R.

\section{Protein-protein co-expression}

152 The linear associations between pairs of proteins expression and estimates of the regression

153 slopes for each group were conducted. This by fitting a linear regression model for each pair

154 of proteins ( 82418 models in total) with expression of one of the proteins as outcome and

155 expression of the other protein, the group and their interaction as independent variables. The

156 intensities were transformed with logarithm of base two before fitting the models and

157 standardized by subtracting mean protein expression and dividing by the standard deviation,

158 to make the slopes comparable between models.

\section{Reproducibility and quality control}

160 The samples were run in random order with the insistency of having samples from each group 161 analyzed in the beginning, middle and the end of the sample batch run. A pooled sample was

162 run every 10th sample as a quality control sample. 18 of the samples were run as duplicates to

163 check for reproducibility. The reliability was evaluated by comparing two injections of the

164 same sample and estimating the agreement using the Bland-Altman method ${ }^{21,22}$. The data was

165 again transformed by the logarithm of base two to stabilize variance.

\section{Pathway analysis}

167 To evaluate common interactions and pathways of the identified proteins with changed levels, 168 the results were further analyzed with IPA software (Ingenuity Systems, Redwood City, CA, 
169 USA, www.ingenuity.com). Differentially expressed proteins were mapped and compared to

170 known pathways, diseases, functions, and connecting regulators using only 406 proteins as a

171 background data set. The data were also evaluated based on upstream regulators with positive

172 z-scores (activating capacity) and negative z-scores (inhibiting capacity). Key regulators are

173 proteins with an earlier established association with the activation or inhibition of many of the

174 identified proteins, but the regulators are not necessarily detected themselves in our MS

175 analysis. Default settings were used except for species, which was set to human. In addition,

176 only experimentally observed relationships were considered. The software was unable to map

177 P69905 protein (Hemoglobin subunit alpha). This protein was therefore not further included

178 in the pathway analysis.

179 Validation of mass spectrometry results

180 Thirty-six out of the 406 proteins that were differentially expressed in our MS data were

181 further validated with the Multiplex ${ }^{\circledR}$ cardiometabolic immunoassay panel (Olink

182 Proteomics, Uppsala, Sweden). The synovial fluid samples were diluted 1:1000 before

183 analysis. Processing, output data quality check, and normalization were performed by Olink

184 Proteomics. All data were delivered as Normalized Protein eXpression (NPX) values on a

$185 \log _{2}$ scale. Data values below the level of detection (LOD) were removed from the dataset.

186 Using these data, the main analysis was repeated with linear multilevel models to estimate the

187 differences between the groups in protein expression and relate them to the original results.

\section{Results}

\section{Label free quantification}

190 In total, 715 proteins were detected and relatively quantified in synovial fluid samples from

19131 subjects. After selection of proteins with limited missing values, 474 proteins were eligible 
192 for the clustering and statistical analysis for comparing the late-stage OA vs control group and

193406 proteins for comparing all 3 groups.

\section{Clustering}

195 Principal component analysis revealed an unsupervised difference between the individual

196 samples clustering the samples very well in their different groups (Fig 1A.). The protein

197 profile of early-stage OA synovial fluid samples clustered between control and late-stage OA

198 samples. The biplot shows some of the proteins that drive this cluster separation (Fig. 1B).

199 Among these are Chemokine (C-X-C motif) ligand 7 (CXCL7), Decorin (PGS2), S10A8,

200 Zinc fingers and homeoboxes protein 3 (ZHX3).

\section{Differentially expressed proteins}

202 Out of 406 proteins analyzed, different patterns of protein expression were identified with

203 over than 200 proteins that where differently expressed between the different groups

204 (Supplemental Fig. S1 and Table S1). Among them 27 proteins (whereof 16 downregulated)

205 with a fold change of 2 or larger in the late-stage OA group compared to control, or in the

206 early-stage OA group compared to control where identified (Fig 2). The most pronounced

207 differences in comparisons with controls were found for PGS2 (fold-change 0.05 with a 95\%

208 CI [0.03-0.09] early-stage OA, 0.03 [0.02-0.06] late-stage OA), CXCL7 (0.091 [0.05-0.17]

209 early-stage OA, 0.061 [0.03-0.11] late-stage OA). Glutathione peroxidase 3 (GPX3) (8.85

210 [4.95-15.8] early-stage OA, 5.91 [3.29-10.63] late-stage OA) and Histidine-rich glycoprotein

211 (HRG) (7.03[3.95-12.51] early-stage OA, 7.79 [4.35-13.95] late-stage OA), where >1 fold

212 changes indicate higher levels in early-stage OA patients than in controls, and vice versa.

213 Inspecting the gradient from the lowest to the highest fold changes in differently expressed

214 proteins (Supplemental Fig. S1), the majority of the proteins that differed between the late-

215 stage OA and control cases were higher in the control samples in comparison to the late-stage

216 OA samples. This observation was even more evident when only comparing the late-stage OA 
217 and control samples. When comparing late-stage OA and early-stage OA samples, only 3

218 proteins were higher in late-stage OA in comparison to early-stage OA - Fibronectin (FINC),

2191.99 [1.04-3.83], hemoglobin subunit alpha (HBA), 2.25 [1.17-4.32], Cartilage acidic protein

2201 (CRAC1), 1.96 [1.02-3.78], whereas 112 proteins were higher in early-stage OA than in

221 late-stage OA samples. Together, all these results suggest an overall decrease in protein

222 expression in late-stage OA.

\section{Biological relevance}

\section{Canonical pathways}

225 Pathway analysis revealed that the differentially expressed proteins were connected to

226 multiple canonical pathways (Table 1). The z-scores suggest whether the pathways are

227 activated or inhibited in accordance to the fold changes of the connected proteins.

228

229

230

231

232

233

234

235

236

237

\section{Upstream regulators}

Pathway analysis also revealed that the differentially expressed proteins were connected to multiple upstream regulators (Fig. 3). For instance, the transcription factor serum response factor (SRF) was activated in the early-stage OA group in comparison to controls. The analysis also suggested that the transcription factor Y-box-binding protein 1 (YBX1) was activated in the late-stage OA group in comparison to controls.

\section{Molecular functions}

Pathway analysis revealed that the differentially expressed proteins were connected to multiple diseases and molecular functions (Supplemental Fig. S2 and Table S2). The z-scores suggest whether the corresponding functions are activated or inhibited in accordance to the fold changes of the connected proteins. The overall pattern suggested that several molecular functions such as cellular development and assembly are activated in the early-stage OA group. It also suggested that cellular movement is deactivated in the late-stage OA group. 


\section{Inflammation}

242 Pathway analysis suggests that the acute phase pathway is inhibited in the early-stage OA and

243 late-stage OA groups in comparison to the controls (Table 1). Leukocyte extravasation

244 signalling is suggested to be activated in the early-stage OA group compared to controls.

245 Additionally, typical markers for inflammation (e.g. CXCL17) were found to be decreased in

246 the early-stage OA and late-stage OA cases in comparison to the controls (Supplemental

247 Table S1).

248 Lipid Metabolism

249 The pathway analysis suggested a strong activation of the LXR/RXR lipid metabolism

250 pathway in both early-stage OA and late-stage OA groups, with a higher evidence of

251 activation for early-stage OA patients (Table 1).

252

253

254

255

256

257

258

259

260

261

262

263

264

Extracellular matrix (ECM) Organization and damage associated molecular patterns DAMPs

The results revealed multiple ECM proteins that were differentially expressed in the synovial fluid when comparing the different groups to each other (Supplementarl Fig. S1 and Table S1). The results further suggested a cluster of collagens differing between the different groups. CO18A1 (fold-change 0.60 and 95\% CI [0.34-1.07] early-stage OA, 0.38 [0.21-0.68] late-stage OA) and CO6A2 (0.47 [0.26-0.85] early-stage OA, 0.53 [0.30-0.96] late-stage OA) had a lower expression in both early-stage OA and late-stage OA cases in comparison to the controls, while CO5A1(3.36 [1.89-5.98] early-stage OA, 2.25 [1.26-4.03] late stage OA) had a higher expression in both early-stage OA and late stage OA cases. CO3A1(0.22 [0.110.42]), CO14A1(0.34 [0.17-0.68]) and CO1A1 (0.18 [0.10-0.35]) all had a lower differential expression in the late-stage OA cases compared to early-stage OA cases. Some DAMP proteins did also express differential expression between the different groups, fibrinogen (gamma, beta and alpha) were lower in OA patients in comparison to controls. Tenascin 
265 (TENA) (2.12 [1.19-3.77] early-stage OA, 2.13 [1.19-3.82] late-stage OA) was higher in OA

266 patients in comparison to controls.

267 Serine protease activity

268 Differentially expressed proteins that were higher in OA patients in comparison to controls

269 had serine-type peptidase activity; among them were MMP2 (fold-change 1,91 and 95\% CI

270 [1.07-3.40] early-stage OA, 1.79 [1.00-3.20] late-stage OA) and MMP3 (2.38 [1.33-4.24]

271 early-stage OA, 2.08 [1.16-3.72] late-stage OA).

\section{Associations between pairs of proteins}

273 Protein co-expression data revealed that the control samples have protein pairs that are both

274 positively and negatively co-expressed (mean slope, 0.29 ), whereas the early-stage OA co-

275 expression revealed an increase in positive co-expression of the same protein pairs (mean

276 slope, 0.48) (Fig 4). The same analysis with late stage OA samples indicated that co-

277 expression between the proteins in these pairs was largely lost at this stage of the disease

278 (mean slope, 0.05). The same pattern could be detected when comparing protein co-

279 expression in all 406 proteins, or when only selecting the differentially expressed proteins in

280 one of the group comparisons but not the other, or even in proteins that did not show

281 differential expression between any of the different groups (Supplemental Fig. S3, Fig. S4 and 282 Fig.S5).

\section{Data quality}

284 Repeatability coefficients estimated using the Bland-Altman approach for the 406 proteins

285 included in the main analysis (i.e. the size of the difference between two measurements of the

286 same sample) had (on a $\log _{2}$ scale) a median of 0.46 , which corresponds to a fold change of

287 1.37. $75 \%$ of the proteins had a repeatability coefficient lower than 0.83 (corresponding to a

288 fold change of 1.77). 


\section{Validation of mass spectrometry label free quantification with Olink}

290 Validating 36 differentially expressed proteins from the MS analysis with immuno-based

291 proximity extension assay from Olink, a similar fold change pattern was found as in the label

292 free MS results (Fig 5). Overall, the Olink data resulted in slightly higher fold changes than

293 MS data. Olink data also had an overall lower dynamic range in comparison to the MS data.

\section{Discussion}

295 The results of this study generated evidence of a profound difference in the global protein

296 profile of knee synovial fluid from controls compared to knee OA patients with more than

297200 proteins differentially expressed between the different groups. Most of the proteins

298 estimated to have a larger than 2-fold change between late-stage OA vs. controls and early-

299 stage OA vs. controls were remarkably similar, suggesting their potential involvement in the

300 OA disease process. The observed protein fold change patterns indicated an overall higher

301 protein expression in early-stage OA, and a reduced global response in late-stage OA.

302 Additionally, evaluation of the co-expression patterns of proteins within each of the three

303 groups indicated that positive co-expression between proteins was increased in early-stage

304 OA compared to controls, and that this co-expression was mostly lost in late-stage OA. The

305 differential expression data revealed that similar pathways were detected to be activated or

306 inhibited in the early stages OA and late stages OA in comparison to the controls. This

307 indicates that the same types of mechanisms might be induced for degenerative meniscal tears

308 as for late-stage OA.

309 The data were analysed both with an unsupervised dimensionality reduction test, PCA and

310 through linear multilevel model ${ }^{23-25}$. A lot of the proteins that were differentially expressed in

311 the statistical model were also among the principal components separating the different

312 samples from each other, resulting in separated clusters in-between the different groups. 
313 Among the 27 proteins that were differentially expressed with a fold change larger than 2

314 have previously been reported to have a connection to osteoarthritis disease progression (Fig

315 4) ${ }^{13,26-31}$. Proteins like cartilage intermediate layer protein 1 (CILP), aggrecan core protein

316 (ACAN, PGCA), decorin (DCN, PGS2) and collagen type I (COL1A1) have been identified

317 in multiple studies to be associated with OA. CILP, can act as an antagonist to TGFB1

318 mediated induction of cartilage matrix genes and IGF1-induced proliferation ${ }^{32}$. Its

319 overexpression may lead to impaired chondrocyte growth and matrix repair. The proteoglycan

320 aggrecan (ACAN or PGCA) is a major component of the ECM of cartilaginous tissues ${ }^{33}$. The

321 major function of this protein is to resist compression in cartilage. It binds avidly to

322 hyaluronic acid and forms negatively charged proteoglycan aggregates that attract water.

323 Decorin (DCN) can affect the rate of collagen type I fibril formation, influencing the

324 crosslinking of the ECM ${ }^{34}$. These proteins exists in the molecular organization of normal

325 articular cartilage and are present in the matrix surrounding chondrocytes ${ }^{35}$. In this study, all

326 these three proteins were decreased in the synovial fluid of both early and late-stage OA in

327 comparison to the controls. Periostin (POSTN) is associated with reparative processes

328 involving cell adhesion and migration ${ }^{36}$. It enhances incorporation of BMP1 in the

329 fibronectin matrix of connective tissues, and subsequent proteolytic activation of lysyl

330 oxidase for the crosslinking of the ECM. In this study, this protein was increased in both early

331 and the late-stage OA in comparison to controls, and there was a 3-fold difference in its levels

332 when comparing early and late-stage OA. Collagen type I (CO1A1) was found to be increased

333 in the synovial fluid in early-stage OA but not in the late-stage. Additionally, higher

334 expression of different types of collagens in early OA compared to the donor group, which

335 might be an indicator of either ECM synthesis or degradation reflected in the synovial fluid.

336 Among these, collagen type III (CO3A1) was increased in early OA but decreased in late-

337 stage OA, compared to controls. Collagen III is known to increase during regeneration and 
338 wound healing ${ }^{37}$, suggesting an attempted regeneration process in early OA. Taken together,

339 the changes in these proteins suggest altered proliferation and development of chondrocytes,

340 as well as widespread changes in the ECM especially in the early-stage of OA.

341 Some of the proteins that have a 2-fold or higher change in differential expression in OA

342 patients compared to controls have previously not been reported to be associated with OA

343 disease progression, e.g. ZHX3 and lymphatic vessel endothelial hyaluronic acid receptor 1

344 (LYVE1). ZHX3 acts as a transcriptional repressor involved in the early stages of

345 mesenchymal stem cell osteogenic differentiation, which plays a fundamental role in skeletal

346 tissue homeostasis ${ }^{38}$. LYVE1 plays a role in autocrine regulation of cell growth and as a

347 hyaluronan transporter, either mediating its uptake for catabolism within lymphatic

348 endothelial cells themselves, or its transport into the lumen of afferent lymphatic vessels for

349 subsequent re-uptake and degradation in lymph nodes ${ }^{39,40}$. ZHX3 was increased in both early

350 and late-stage OA while LYVE1 was decreased in the OA patients in comparison to the

351 controls. The positive co-expression of these two proteins seen in controls and in early-stage

352 OA, compared to the rest of the 27 differentially expressed proteins, seemed to be diminished

353 in late-stage OA, suggesting that the crosstalk between these proteins is lost. This data could

354 reflect a dysfunction in skeletal tissue homeostasis in late-stage OA.

355 Among the proteins that were found to be exclusively upregulated in late-stage OA was

356 CRTAC1, a glycosylated ECM protein that can be found in the deep zone of articular

357 cartilage ${ }^{41,42}$. This protein is used as a marker to distinguish chondrocytes from osteoblasts

358 and mesenchymal stem cells in culture. It can be detected in cartilage, bone, and cultured

359 chondrocytes, but not in osteoblasts. The altered expression of this protein in the synovial

360 fluid samples from late-stage OA cases might be a reflection of the severely damaged knee. It

361 could also reflect higher exposure of the synovial fluid to the deep zone of articular cartilage. 
362 The transcription factors that were mapped through the differentially expressed proteins to be

363 activated in the different OA stages were SRF and YBX1. SRF is involved in differentiation,

364 proliferation and cell cycle progression ${ }^{43}$. This suggests increased cellular activity in early-

365 stage OA. YBX1 is involved in a number of cellular processes including proliferation,

366 differentiation, and the cellular stress response ${ }^{44,45}$. YBX1 activation in late-stage OA

367 indicates the activation of responses for cell survival under stress conditions.

369 The degree of which inflammation contributes to OA pathogenesis and progression is an

370 ongoing discussion in the field. There is still controversy whether it is predominantly an

371 inflammatory, a low-grade inflammatory or a "wear and tear" mechanical driven disease ${ }^{46-48}$.

372 In this study, we have utilized discovery based MS which is capable of capturing a high

373 dynamic range of molecules in a sample and we could not find a strong evidence of a typical

374 inflammatory response associated with OA. On the other hand, this analysis may not detect

375 the very low abundant inflammatory markers if no extensive fractionation is carried out. Well

376 known inflammatory signalling molecules were only detected in low percentage, and the

377 differential expression among disease stages of such molecules did not indicate that

378 inflammation as the driving force in the OA pathogenesis. Previous studies supporting

379 inflammation as a driving force of OA were not discovery based and only explored specific

380 inflammatory molecules in bio fluids such as blood or plasma. ${ }^{46}$ The lack of a robust

381 inflammatory signature in our findings matches previous reports using discovery based

382 proteomics ${ }^{13,15-17}$. Furthermore, clinical trials that targeted inflammation such as IL

383 antagonist therapies and anti-cytokine therapeutic drugs failed to reverse $\mathrm{OA}^{49}$. Although

384 inflammatory molecules could be detected even at a low level, the degree of which they

385 contribute to disease progression remains unknown. 
386 Overall, these results show that a higher proportion of the detected proteins were upregulated

387 in early-stage OA while late-stage OA was characterized by an imbalance of the homeostasis

388 of the synovial fluid proteome. Furthermore, our data indicates that profiling the proteomic

389 signature of a disease state may not be solely sufficient to elucidate the underlying

390 mechanisms of OA. However, the disrupted cross talk between proteins across different

391 stages of the disease might provide insights into the global mechanisms of disease onset and

392 progression.

393 We would like to acknowledge some important limitations of this study. Four out of seven

394 synovial fluid samples in the early-stage OA group were diluted during aspiration. To

395 compensate for this, their total protein contents were normalized, leaving us with reduced

396 individual variation detected in the differential expression analysis. On the other hand, the co-

397 expression data were based on pairwise comparisons between proteins within the same

398 sample, which is a major strength in this study. This minimizes any type of variation that

399 could have come from the sample preparation or analysis. For the early-stage OA group, the

400 same pattern of an overall increase in protein expression was detected in both the differential

401 expression analysis and the co-expression analysis.

402 The samples used as controls was from post mortem donors. To our knowledge there is no

403 study of how quickly death effects the proteome in synovial fluid. Forensic studies have

404 investigated the change of specific biochemical like potassium, glucose and creatinine ${ }^{50}$.

405 Even though only very few metabolites showed a correlation between the concentration and

406 time post-mortem the countable difference was only detected after $120 \mathrm{~h} .^{50,51}$. In this study,

407 all samples were obtained within $48 \mathrm{~h}$ post-mortem, and the specimens were frozen at $-80^{\circ} \mathrm{C}$

408 within $2 \mathrm{~h}$ of extraction to be eligible as controls. Therefore, the proteomic status was

409 assumed to be stable post-mortem.

410 


\section{Conclusions}

412 We found profound differences in the protein profiles of synovial fluid from controls and

413 patients with different stages of OA. The synovial fluid of early-stage OA might represent a

414 "raging battle field" of increased protein activity, while late-stage OA displays the

415 "aftermath". Further efforts should be made to elucidate the early processes in the disease,

416 which have the greatest potential to be modified by therapeutic interventions.

\section{Acknowledgements}

418 This work was supported by the European Research Council (ERC) under the European

419 Union's Horizon 2020 research and innovation programme (grant agreement \#771121), the

420 Swedish Research Council, the Foundation for Research in Rheumatology (FOREUM), the

421 IAB Lundberg Foundation, the Greta and Johan Kock Foundation, the Swedish Rheumatism

422 Association, the Österlund Foundation, the Governmental Funding of Clinical Research

423 program within the National Health Service (ALF), the Royal Physiographic Society of Lund

424 and the Faculty of Medicine, Lund University, Sweden. The funders had no role in study

425 design, data collection and analysis, decision to publish, or preparation of the manuscript.

\section{Author contributions}

428 Conception and design: NA, ME, and PÖ. Provision of study materials and tissue preparation:

429 ME, NA, EF, VH, PÖ, JT, PN. Mass spectrometry analysis: NA. Statistical analysis: AT and

430 NA. Interpretation of results: All co-authors. Drafting of the article: NA. Critical revision of

431 the article for important intellectual content: NA, ME, AT, VH, PÖ and JT. Final approval of

432 the article: All co-authors

\section{Competing interests}


435 The authors declare no competing interests.

436

\section{References}

$4381 \quad$ Wittenauer, R., Smith, L. \& Aden, K. Background paper 6.12 osteoarthritis. World $439 \quad$ Health Organisation (2013).

4402 Loeser, R. F., Goldring, S. R., Scanzello, C. R. \& Goldring, M. B. Osteoarthritis: A

441

442

443

444

445

446

447

448

449

450

451

452

453

454

455

456

457

458

459

460

461

462

463

464

465

466

467

468

469

470

471

472

473

474

475

476

477

478

479

480 disease of the joint as an organ. Arthritis \& Rheumatism 64, 1697-1707, doi:10.1002/art.34453 (2012).

3 Loeser, R. F. The role of aging in the development of osteoarthritis. Transactions of the American Clinical and Climatological Association 128, 44 (2017).

4 Shane Anderson, A. \& Loeser, R. F. Why is osteoarthritis an age-related disease? Best Pract Res Clin Rheumatol 24, 15-26, doi:10.1016/j.berh.2009.08.006 (2010).

5 Dieppe, P. A. \& Lohmander, L. S. Pathogenesis and management of pain in osteoarthritis. The Lancet 365, 965-973 (2005).

6 Braun, H. J. \& Gold, G. E. Diagnosis of osteoarthritis: imaging. Bone 51, 278-288 (2012).

7 Hunter, D. J., Nevitt, M., Losina, E. \& Kraus, V. Biomarkers for osteoarthritis: current position and steps towards further validation. Best Pract Res Clin Rheumatol 28, 6171, doi:10.1016/j.berh.2014.01.007 (2014).

8 Attur, M., Krasnokutsky-Samuels, S., Samuels, J. \& Abramson, S. B. Prognostic biomarkers in osteoarthritis. Current opinion in rheumatology 25, 136 (2013).

9 Watt, F. E. Osteoarthritis biomarkers: year in review. Osteoarthritis and cartilage 26, 312-318, doi:10.1016/j.joca.2017.10.016 (2018).

10 Mayeux, R. Biomarkers: potential uses and limitations. NeuroRx 1, 182-188 (2004).

11 Ball, J. R. \& Micheel, C. M. Evaluation of biomarkers and surrogate endpoints in chronic disease. (National Academies Press, 2010).

12 Sharma, A. R., Jagga, S., Lee, S. S. \& Nam, J. S. Interplay between cartilage and subchondral bone contributing to pathogenesis of osteoarthritis. Int J Mol Sci 14, 19805-19830, doi:10.3390/ijms141019805 (2013).

13 Balakrishnan, L. et al. Proteomic analysis of human osteoarthritis synovial fluid. Clin Proteomics 11, 6, doi:10.1186/1559-0275-11-6 (2014).

14 Peffers, M. J., Smagul, A. \& Anderson, J. R. Proteomic analysis of synovial fluid: current and potential uses to improve clinical outcomes. Expert Rev Proteomics 16, 287-302, doi:10.1080/14789450.2019.1578214 (2019).

15 Liao, W. X. et al. Proteomic analysis of synovial fluid in osteoarthritis using SWATHmass spectrometry. Mol Med Rep 17, 2827-2836, doi:10.3892/mmr.2017.8250 (2018).

16 Brophy, R. et al. Proteomic analysis of synovial fluid identifies periostin as a biomarker for anterior cruciate ligament injury. Osteoarthritis and cartilage 27, 17781789 (2019).

17 Ritter, S. Y. et al. Proteomic analysis of synovial fluid from the osteoarthritic knee: comparison with transcriptome analyses of joint tissues. Arthritis Rheum 65, 981-992, doi:10.1002/art.37823 (2013).

18 Ruiz-Romero, C., Rego-Perez, I. \& Blanco, F. J. What did we learn from 'omics' studies in osteoarthritis. Current Opinion in Rheumatology 30 (2018).

19 Tsezou, A. Osteoarthritis year in review 2014: genetics and genomics. Osteoarthritis and cartilage 22, 2017-2024 (2014). 
$53140 \quad$ Lawrance, W., Banerji, S., Day, A. J., Bhattacharjee, S. \& Jackson, D. G. Binding of

532

533

534

535

536

537

538

539

540

541

542

543

544

545

546

547

548

549

550

551

552

553

554

555

556

557

558

559

560

561

562

563

564

565

566

567

568

569

570

571

572

573

574

575 hyaluronan to the native lymphatic vessel endothelial receptor LYVE-1 is critically dependent on receptor clustering and hyaluronan organization. Journal of Biological Chemistry 291, 8014-8030 (2016).

41 Steck, E. et al. Chondrocyte expressed protein-68 (CEP-68), a novel human marker gene for cultured chondrocytes. Biochem J 353, 169-174, doi:10.1042/02646021:3530169 (2001).

42 Steck, E. et al. Chondrocyte secreted CRTAC1: a glycosylated extracellular matrix molecule of human articular cartilage. Matrix Biol 26, 30-41, doi:10.1016/j.matbio.2006.09.006 (2007).

43 Schratt, G. et al. Serum Response Factor Is Required for Immediate-Early Gene Activation yet Is Dispensable for Proliferation of Embryonic Stem Cells. Molecular and Cellular Biology 21, 2933-2943, doi:10.1128/mcb.21.8.2933-2943.2001 (2001).

44 Kwon, E. et al. The RNA-binding protein YBX1 regulates epidermal progenitors at a posttranscriptional level. Nat Commun 9, 1734, doi:10.1038/s41467-018-04092-0 (2018).

45 Eliseeva, I. A., Kim, E. R., Guryanov, S. G., Ovchinnikov, L. P. \& Lyabin, D. N. Ybox-binding protein 1 (YB-1) and its functions. Biochemistry (Moscow) 76, 14021433, doi:10.1134/S0006297911130049 (2011).

46 Chow, Y. Y. \& Chin, K. Y. The Role of Inflammation in the Pathogenesis of Osteoarthritis. Mediators Inflamm 2020, 8293921, doi:10.1155/2020/8293921 (2020).

47 de Lange-Brokaar, B. J. E. et al. Synovial inflammation, immune cells and their cytokines in osteoarthritis: a review. Osteoarthritis and cartilage 20, 1484-1499, doi:https://doi.org/10.1016/j.joca.2012.08.027 (2012).

48 Robinson, W. H. et al. Low-grade inflammation as a key mediator of the pathogenesis of osteoarthritis. Nat Rev Rheumatol 12, 580-592, doi:10.1038/nrrheum.2016.136 (2016).

49 Haseeb, A. \& Haqqi, T. M. Immunopathogenesis of osteoarthritis. Clin Immunol 146, 185-196, doi:10.1016/j.clim.2012.12.011 (2013).

50 Madea, B., Kreuser, C. \& Banaschak, S. Postmortem biochemical examination of synovial fluid--a preliminary study. Forensic Sci Int 118, 29-35, doi:10.1016/s03790738(00)00372-8 (2001).

51 Holley, H. L., Patton, F. M., Pigman, W. \& Platt, D. An electrophoretic study of normal and post-mortem human and bovine synovial fluids. Arch Biochem Biophys 64, 152-163, doi:10.1016/0003-9861(56)90251-x (1956). 
576 Figures

577

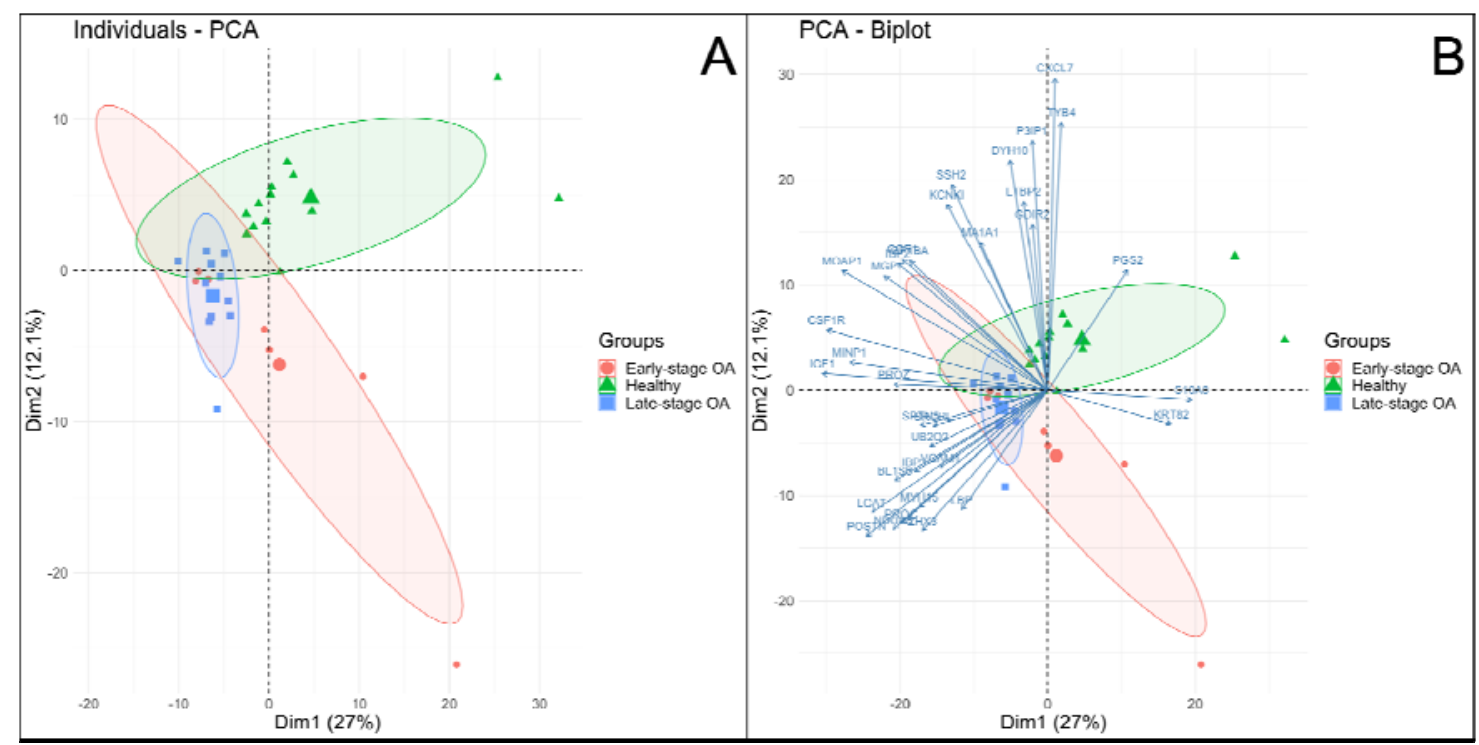

578 Fig 1. PCA and Biplot; 406 proteins from the label free quantification were used to

579 cluster the different samples. A) Principal component analysis of each sample in

580 each group driven by the label free quantification of the 406 proteins. B) Biplot

581 showing the proteins that drive the separation of the clusters. 


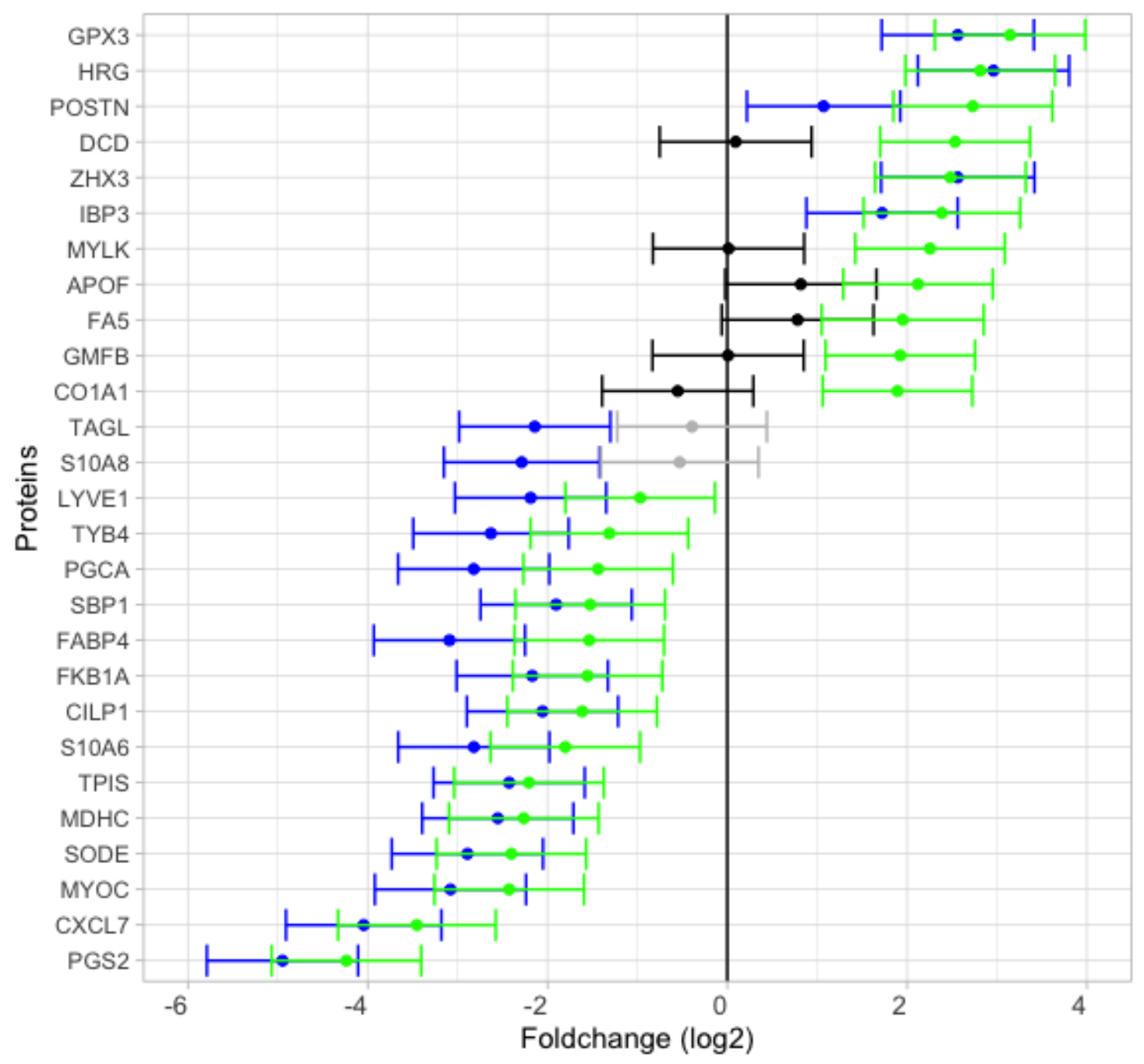

582

583 Fig 2. Differentially expressed proteins with an estimated fold change larger

584 than 2. Comparison between early-stage OA vs controls (green and gray bars),

585 Comparison between late-stage OA vs controls (blue and black bars). Blue and green bars represent differentially expressed proteins whose $95 \%$ Cls exclude 0. 
588

A

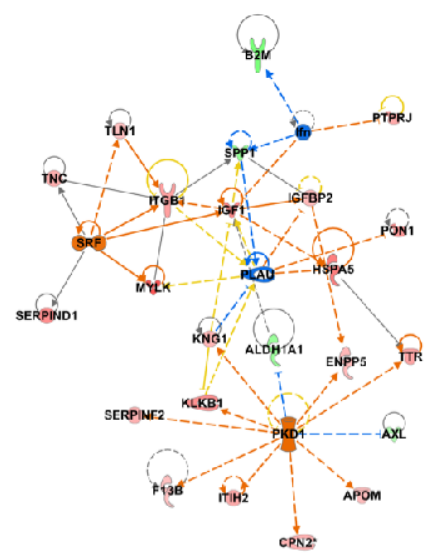

B

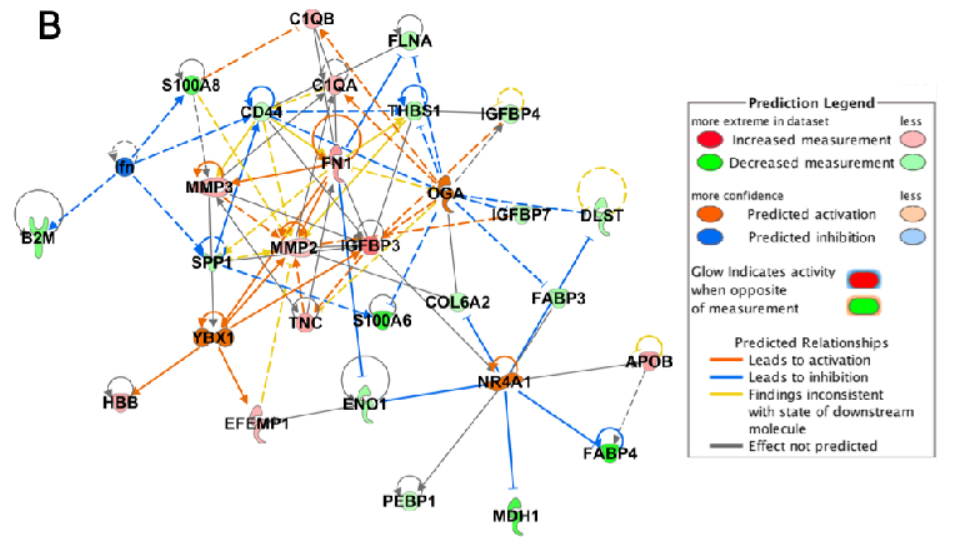

589 Fig 3. The network of upstream regulators. A) Upstream regulators suggested to

590 be activated or deactivated by the differentially expressed proteins in the early-stage

591 OA group: Ifn, PKD1, PLAU and SRF. B) Upstream regulators suggested to be

592 activated or deactivated from the differentially expressed proteins in the late-stage

593 OA group: Ifn, NR4A1, OGA, YBX1 
Differentially expressed proteins with an estimated fold change larger than 2

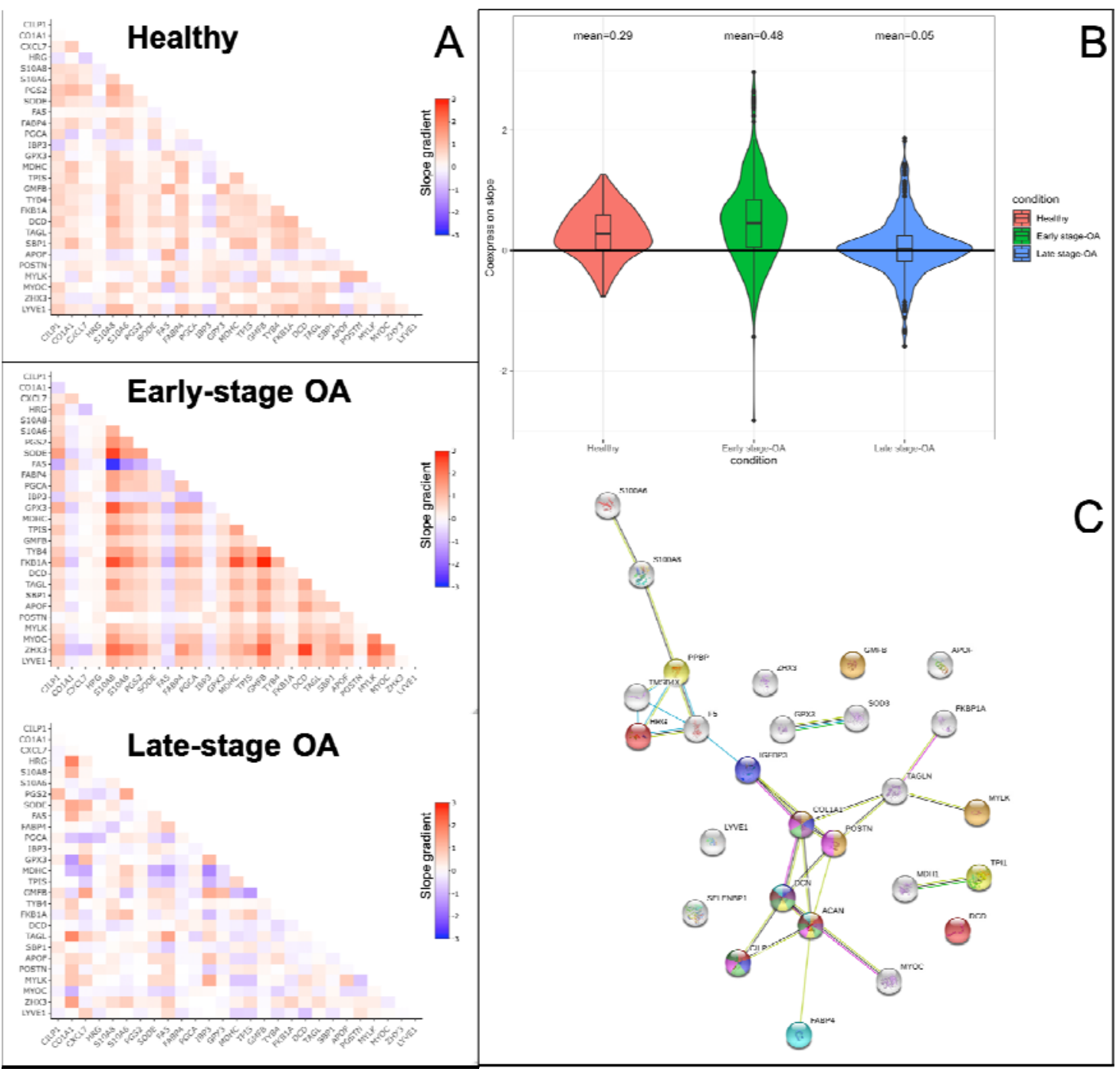

Fig 5. The estimated slope for each protein-protein co-expression pair between

596 the 27 differentially expressed proteins with an estimated fold change larger

597 than 2. A) The estimated slopes for each protein-protein co-expression pair of the 27

598 differentially expressed proteins with an estimated fold change larger than 2 are

599 represented on the $x$ and $y$ axes for a for each group. B) The distribution of the

600 estimated slopes for each of the protein-protein co-expression and the mean slope

601 value is represented in the violin plot for each group. C) The protein-protein

602 interaction network for the 27 proteins that were estimated to have a fold change

603 larger than 2. Each color represents a previous study that suggested these proteins

604 to be involved in osteoarthritis. 


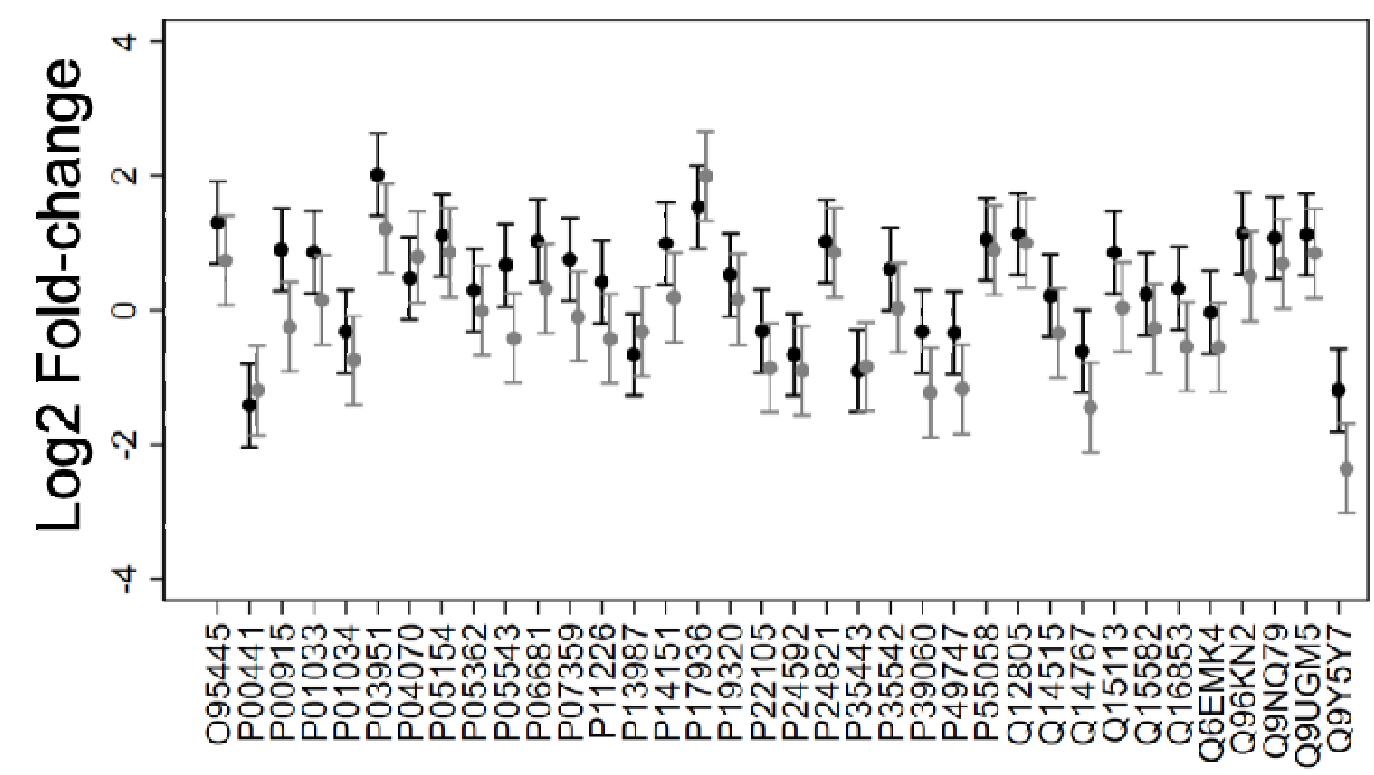

- Olink - DIA

606

607 Fig 6. Validation of MS data. Comparison of fold changes for the late-stage OA vs 608 controls between the two methods used, DIA and Olink, shown as differences with $60995 \%$ Cls. 
611 score of activation if positive and deactivation if negative.

\begin{tabular}{|c|c|c|}
\hline Ingenuity Canonical Pathways & zScore & Molecules \\
\hline LXR/RXR Activation & 2,32 & $\begin{array}{l}\text { AGT,AHSG,ALB,APOB,APOC1,APOF,APOM,C4A/C4B, } \\
\text { FGA,KNG1,LBP,ORM1,PON1,SERPINF2,TTR }\end{array}$ \\
\hline $\begin{array}{l}\text { Neuroprotective Role of THOP1 in Alzheimer's } \\
\text { Disease }\end{array}$ & 2,24 & AGT,C1R,F11,HGFAC,HTRA1,KNG1,MASP1,YWHAE \\
\hline $\begin{array}{l}\text { Production of Nitric Oxide and Reactive Oxygen } \\
\text { Species in Macrophages }\end{array}$ & 2,12 & ALB,APOB,APOC1,APOF,APOM,CAT,ORM1,PON1 \\
\hline Unfolded protein response & 2,00 & HSPA2,HSPA5,HSPA8,PDIA6 \\
\hline Hepatic Fibrosis Signaling Pathway & 1,63 & AGT,COL1A1,COL3A1,ITGB1,MYLK,SPP1 \\
\hline Intrinsic Prothrombin Activation Pathway & 1,15 & $\begin{array}{l}\text { COL1A1,COL3A1,F10,F11,F13B,F5,F9,FGA,FGB, } \\
\text { FGG,KLKB1,KNG1,PROC,PROS1 }\end{array}$ \\
\hline Leukocyte Extravasation Signaling & 1,00 & CDH5,ITGB1,MMP2,MMP3 \\
\hline
\end{tabular}




\begin{tabular}{|c|c|c|}
\hline Synaptogenesis Signaling Pathway & 1,00 & CDH5,CFL1,HSPA8,TLN1 \\
\hline HOTAIR Regulatory Pathway & 1,00 & COL1A1,MMP2,MMP3,SPP1 \\
\hline Integrin Signaling & 1,00 & ITGB1,MYLK,PFN1,TLN1 \\
\hline BAG2 Signaling Pathway & 1,00 & CTSB,HSPA2,HSPA5,HSPA8 \\
\hline 3-phosphoinositide Biosynthesis & 1,00 & ACP1,CILP,MINPP1,PTPRJ \\
\hline 3-phosphoinositide Degradation & 1,00 & ACP1,CILP,MINPP1,PTPRJ \\
\hline $\begin{array}{l}\text { D-myo-inositol }(1,4,5,6) \text {-Tetrakisphosphate } \\
\text { Biosynthesis }\end{array}$ & 1,00 & ACP1,CILP,MINPP1,PTPRJ \\
\hline $\begin{array}{l}\text { D-myo-inositol }(3,4,5,6) \text {-tetrakisphosphate } \\
\text { Biosynthesis }\end{array}$ & 1,00 & ACP1,CILP,MINPP1,PTPRJ \\
\hline D-myo-inositol-5-phosphate Metabolism & 1,00 & ACP1,CILP,MINPP1,PTPRJ \\
\hline Superpathway of Inositol Phosphate Compounds & 1,00 & ACP1,CILP,MINPP1,PTPRJ \\
\hline Osteoarthritis Pathway & 0,45 & ACAN,DCN,HTRA1,ITGB1,MMP3,SPP1 \\
\hline
\end{tabular}




\begin{tabular}{|c|c|c|}
\hline Sirtuin Signaling Pathway & 0,45 & LDHA,LDHB,NDUFA8,SOD1,SOD3 \\
\hline GP6 Signaling Pathway & 0,00 & COL1A1,COL3A1,COL5A1,COL6A2,FGA,FGB,FGG,TLN1 \\
\hline RhoA Signaling & 0,00 & CFL1,IGF1,MYLK,PFN1 \\
\hline Extrinsic Prothrombin Activation Pathway & 0,00 & F10,F13B,F5,FGA,FGB,FGG,PROC,PROS1 \\
\hline Gluconeogenesis I & $-0,45$ & ALDOC,ENO1,GAPDH,MDH1,MDH2 \\
\hline Coagulation System & $-0,77$ & $\begin{array}{l}\text { F10,F11,F13B,F5,F9,FGA,FGB,FGG,KLKB1,KNG1, } \\
\text { PROC,PROS1,SERPINA5,SERPIND1,SERPINF2 }\end{array}$ \\
\hline Actin Cytoskeleton Signaling & $-0,82$ & $\begin{array}{l}\text { CFL1,ITGB1,KNG1,LBP,MYLK,PFN1,SSH2,TLN1, } \\
\text { TMSB10/TMSB4X }\end{array}$ \\
\hline Glycolysis I & $-1,00$ & ALDOC,ENO1,GAPDH,TPI1 \\
\hline RhoGDI Signaling & $-1,00$ & ARHGDIB,CDH5,CFL1,ITGB1 \\
\hline SPINK1 Pancreatic Cancer Pathway & $-1,00$ & CPN1,CPQ,CTSB,KLKB1 \\
\hline eNOS Signaling & $-1,00$ & HSPA2,HSPA5,HSPA8,KNG1 \\
\hline
\end{tabular}




\begin{tabular}{|c|c|c|}
\hline Acute Phase Response Signaling & $-1,13$ & $\begin{array}{l}\text { AGT,AHSG,ALB,C1R,C4A/C4B,FGA,FGB,FGG,HRG,ITIH2, } \\
\text { KLKB1,LBP,ORM1,SERPIND1,SERPINF2,TTR }\end{array}$ \\
\hline Ingenuity Canonical Pathways & zScore & Molecules \\
\hline LXR/RXR Activation & 1,26 & $\begin{array}{l}\text { AHSG,APOA4,APOB,FGA,KNG1,LBP, } \\
\text { ORM1,PLTP,PON1,S100A8 }\end{array}$ \\
\hline Sirtuin Signaling Pathway & 1,00 & LDHA,LDHB,SOD1,SOD3 \\
\hline HIPPO signaling & 1,00 & CD44,YWHAE,YWHAG,YWHAZ \\
\hline $\begin{array}{l}\text { Production of Nitric Oxide and Reactive Oxygen } \\
\text { Species in Macrophages }\end{array}$ & 0,45 & APOA4,APOB,ORM1,PON1,S100A8 \\
\hline Osteoarthritis Pathway & 0,45 & $\begin{array}{l}\text { ACAN,ANXA2,ANXA5,DCN,FN1,HTRA1,MMP3, } \\
\text { S100A8,S100A9,SPP1 }\end{array}$ \\
\hline HOTAIR Regulatory Pathway & 0,00 & CD44,MMP2,MMP3,SPP1 \\
\hline Acute Phase Response Signaling & $-0,38$ & $\begin{array}{l}\text { AHSG,APCS,FGA,FGB,FGG,FN1,HRG,ITIH2, } \\
\text { KLKB1,LBP,ORM1,PLG,SERPIND1 }\end{array}$ \\
\hline Coagulation System & $-0,90$ & $\begin{array}{l}\text { F11,F9,FGA,FGB,FGG,KLKB1,KNG1,PLG,PROS1, } \\
\text { SERPINA5,SERPIND1 }\end{array}$ \\
\hline
\end{tabular}




\begin{tabular}{|l|l|l|}
\hline Intrinsic Prothrombin Activation Pathway & $-1,00$ & $\begin{array}{l}\text { COL18A1,COL3A1,F11,F9,FGA,FGB, } \\
\text { FGG,KLKB1,KNG1,PROS1 }\end{array}$ \\
\hline GP6 Signaling Pathway & $-1,89$ & COL18A1,COL3A1,COL5A1,COL6A2,FGA,FGB,FGG \\
\hline Hepatic Fibrosis Signaling Pathway & $-2,24$ & CCN2,COL18A1,COL3A1,FTH1,SPP1 \\
\hline
\end{tabular}

